\title{
LOCALLY (SOLUBLE-BY-FINITE) GROUPS WITH VARIOUS RESTRICTIONS ON SUBGROUPS OF INFINITE RANK
}

\author{
MARTYN R. DIXON, MARTIN J. EVANS \\ Department of Mathematics, University of Alabama, Tuscaloosa, AL 35487, U.S.A. \\ e-mail:mdixon@gp.as.ua.edu,mevans@gp.as.ua.edu \\ and HOWARD SMITH \\ Department of Mathematics, Bucknell University, Lewisburg, PA 17837, U.S.A. \\ e-mail: howsmith@bucknell.edu
}

(Received 19 July, 2004; accepted 10 January, 2005)

\begin{abstract}
We present some results on locally (soluble-by-finite) groups with various restrictions on subgroups of infinite rank.

2000 Mathematics Subject Classification.
\end{abstract}

1. Introduction. Let $G$ be a group and suppose that every abelian subgroup of $G$ has finite rank. (For $r$ a positive integer, a group $G$ has finite (Prüfer) rank at most $r$ if every finitely generated subgroup of $G$ is generated by $r$ elements; if there is no such $r$ then $G$ has infinite rank.) For certain classes of groups $G$ this suffices to ensure that $G$ itself has finite rank. For example, this is the case for locally finite groups [14], locally nilpotent groups (see [10, Theorem 6.36 and its corollaries]), and for hyperabelian (and even radical) groups [1]. It is not the case for locally soluble groups, as shown in [9]. However, the following result was established in [4].

THEOREM 1. Let $G$ be a locally (soluble-by-finite) group.

(i) If every locally soluble subgroup of $G$ has finite rank then $G$ has finite rank.

(ii) If every abelian subgroup of $G$ has finite rank and there is a positive integer $r$ such that every torsion-free abelian subgroup of $G$ has rank at most $r$ then $G$ has finite rank.

Our proof in [4] of part (i) of Theorem 1 involved an appeal to the Classification Theorem for finite simple groups (CFSG), and one of our objectives in the present article is to provide a CFSG-free proof of this result; for part (ii) one immediately reduces to the case where $G$ itself is locally soluble, using (i), and so no further appeal to CFSG is required. Accordingly, we shall not re-prove part (ii) here. Proposition 1 below is quite straightforward to prove, and constitutes a major component of our revised proof. We remark that our original proof in [4] also involved the establishing of some interesting results on sequences of finite semi-simple groups.

Theorem 1 may be viewed as belonging to a larger family of results stating that a group with "many" subgroups having a certain property $P$, or "few" subgroups not having $P$, itself satisfies $P$. Among the many articles on this broad topic, in addition to those referred to above, we mention here [5], [7] and [13]. For a property $P$ of groups, a group $G$ satisfies the weak maximal condition for non- $P$ subgroups if there is no infinite ascending chain $H_{0}<H_{1}<\ldots$ of non- $P$-subgroups of $G$ with each index $\left|H_{i+1}: H_{i}\right|$ infinite. The weak minimal condition for non- $P$ subgroups is defined similarly. Zaičev 
proved in [16] that a locally (soluble-by-finite) group $G$ satisfying either the weak maximal or the weak minimal condition for all subgroups is a soluble-by-finite minimax group, that is, $G$ has a normal soluble subgroup $H$ of finite index which in turn has a finite normal series whose factors are abelian and satisfy either $\max$ or $\min$. In particular, $G$ has finite rank. Here we establish the following two results.

THEOREM 2. Let $G$ be a locally (soluble-by-finite) group that satisfies the weak minimal condition for locally soluble subgroups of infinite rank. Then $G$ has finite rank.

THEOREM 3. Let $G$ be a locally (soluble-by-finite) group that satisfies the weak maximal condition for locally soluble subgroups of infinite rank. Then $G$ has finite rank.

On the way to proving Theorems 2 and 3 we shall establish a further result, presented as Theorem 4 below. Firstly we require some more notation. A locally (nilpotent-by-finite) group $G$ is (here) said to have finite 0-rank if there is an integer $r$ such that the rank of every finitely generated torsion-free nilpotent subgroup of $G$ is at most $r$; otherwise $G$ has infinite 0 -rank. Also, for a subgroup $X$ of a group $G$, we say that the isolator of $X$ in $G$ is $G$, written $I_{G}(X)=G$, if every element of $G$ has a nonzero power in $X$. We may now state our result.

THEOREM 4. Let $G$ be a locally (nilpotent-by-finite) group that has infinite 0-rank. Then there are subgroups $H$ and $K$ of $G$, each of infinite 0-rank, and infinite chains $H=H_{0}<H_{1}<\ldots$ and $K=K_{0}>K_{1}>\ldots$ of subgroups of $G$ such that each of the indices $\left|H_{i+1}: H_{i}\right|,\left|K_{i}: K_{i+1}\right|$ is infinite. If $G$ is countable then $H$ and $K$ may be chosen so that $I_{G}(H)=G=I_{G}(K)$.

A further avenue of investigation, and one pursued in (for example) [2], [12] and [13], is the consideration of groups $G$ in which there are "few" conjugacy classes of non$P$ subgroups, one possible conclusion being that there are no such subgroups! Along these lines, and using the contrapositive form of the implication suggested above, we have the last of our main results.

THEOREM 5. Let $G$ be a locally (soluble-by-finite) group and suppose that $G$ has infinite rank. Then $G$ has infinitely many subgroups of infinite rank that are pairwise non-conjugate.

Next, we present two results that are slightly stronger than we need and that will serve to isolate a couple of steps in the subsequent argument. As usual, $\gamma_{i}(X)$ denotes the $i t h$ term of the lower central series of a group $X$, and the Fitting radical Fitt $(X)$ of $X$ is the product of all normal nilpotent subgroups of $X$.

Proposition 1. Let $F$ be a finitely generated group, $T$ the maximal normal torsion subgroup of $F$, and let $H / T=F i t t(F / T)$. Suppose that $F / H$ has a normal torsion-free abelian subgroup $K / H$ of finite index, and that $K / T$ has finite rank at most $r$. Then there is a positive integer $m$ depending only on $r$ such that $\gamma_{r+1}\left(\left(\left(F^{m}\right)^{\prime}\right)^{m}\right)$ is periodic. Consequently, if $G$ is a group in which every finitely generated subgroup $F$ has the structure described above (where $r$ is fixed) then $\gamma_{r+1}\left(\left(\left(G^{m}\right)^{\prime}\right)^{m}\right)$ is periodic. (Thus $G$ is "periodic-by-(nilpotent of class at most $r$ )-by-(exponent dividing $m$ )-by-abelian-by-(exponent dividing $m)$.")

PROPOSITION 2. Let $G$ be a finitely generated periodic-by-soluble group, with maximal normal periodic subgroup $T$, let $H / T=\operatorname{Fit}(G / T)$ and suppose that $G / T$ has finite rank.

(i) If $H / T$ has rank $r$ then there is a positive integer d depending only on $r$ such that $G / T$ has derived length at most $d$. 
(ii) If $G / H$ is (free abelian of rank $s$ )-by-finite then there is a positive integer $m$ depending only on s and a normal subgroup $C$ of index at most $m$ in $G$ such that $H C^{\prime} / H$ is finite.

Finally in this section we summarize some well-known results related to groups of finite rank, results that we will have occasion to use in the subsequent section, often without further reference.

(a) A finitely generated soluble group of finite rank is minimax [10, Theorem 10.38] and hence nilpotent-by-abelian-by-finite [10, Theorem 3.25].

(b) A group of finite rank is locally soluble if and only if it has a characteristic ascending abelian series [10, Lemma 10.39 and Theorem 10.38].

(c) A locally finite group of finite rank is almost locally soluble, that is, it has a normal locally soluble subgroup of finite index [14]. Indeed, the same holds for locally (soluble-by-finite) groups, by [3] (see also [4]).

2. Proofs. We begin by establishing the auxiliary results stated in the introduction. It will be evident that we have made no attempt to obtain "best possible" bounds.

Proof of Proposition 1. First we note that, since the claim is that the defined verbal subgroup of $G$ is periodic, we may as well assume that $G=F$. By $[\mathbf{1 5}, 9.33]$ there is an integer $m=m(r)$ such that every finite subgroup of $G L(r, Q)$ has order at most $m$, and it follows (in particular) that $G^{m}$ centralizes $K / H$, so $G^{m} H / H$ is centre-by-finite, from which it follows that $L / H:=\left(G^{m}\right)^{\prime} H / H$ is finite. Now each upper central factor $A$ of $H / T$ is $G$-invariant and torsion-free abelian of rank at most $r$, and as above it follows that $L^{m}$ centralizes every such factor and hence (using the fact that $H / T$ also has nilpotency class at most $r$ ) that $H / T$ is contained in the $r$ th term of the upper central series of $L^{m} H / T$. But this implies that $\gamma_{r+1}\left(L^{m} H / T\right)$ is finite [10, Corollary 2 to Theorem 4.21] and hence trivial. Since $\left(G^{m}\right)^{\prime} \leq L$, the result follows.

Proof of Proposition 2. We may assume that $T=1$, so that $G$ is minimax and hence nilpotent-by-abelian-by-finite. For part (i) we may apply the well-known result of Zassenhaus on (locally) soluble linear groups [10, Theorem 3.23] to deduce that there is a positive integer $d=d(r)$ such that the $d t h$ term $L$ of the derived series of $G$ centralizes each upper central factor of $H$. If $L H / H$ is nontrivial then it has a nontrivial $G$-invariant abelian subgroup $M / H$, but $M$ is nilpotent and hence contained in $H$, so we have a contradiction. Since $H$ has nilpotency class at most $r$ the result follows. For part (ii) we may argue as in the proof of Proposition 1, with $m$ as stated there, to obtain the result.

Proof of Theorem 1(i). We may assume that $G$ is countable and so is the ascending union of finitely generated subgroups $F_{1}<F_{2}<\ldots$. For each $i$ let $S_{i}$ denote the soluble radical of $F_{i}$, and set $R=\left\langle S_{i}: i \geq 1\right\rangle$. Then $R$ is locally soluble and hence of finite rank $r$, say, and so $G$ satisfies the hypotheses and hence the conclusion of Proposition 1. Every abelian subgroup of $G$ has finite rank and so the periodic (and hence locally finite) radical $P$ of $G$ has finite rank and hence has a locally soluble radical $Q$ of finite index. Since the centralizer of $P / Q$ in $G$ has finite index we may assume that $P=Q$. As a locally soluble group of finite rank, $P$ has a characteristic (and hence $G$-invariant) ascending series with abelian factors. We proceed to work our way up the series given by Proposition 1, showing that successive terms have finite rank. Let $M$ be a normal 
subgroup of $G$ such that $P \leq M$ and $\gamma_{r+1}(M) \leq P$. Then $M$ is a hyperabelian group all of whose abelian subgroups have finite rank, and so $M$ has finite rank and is therefore locally soluble. Next, let $L$ be a normal subgroup of $G$ containing $M$ such that $L / M$ has finite exponent. If $S / M$ is a locally soluble subgroup of $L / M$ then $S$ is locally soluble (since finitely generated subgroups of $S$ are finite $\bmod M$ ) and so $S$ and hence $S / M$ has finite rank, giving $L / M$ of finite rank and hence $L$ of finite rank. As before we may pass to an appropriate subgroup of finite index in $L$ and assume that $L$ is locally soluble and hence has an ascending $G$-invariant abelian series. If $N / L$ is a normal abelian subgroup of $G / L$ then $N$ is hyperabelian and therefore of finite rank. Finally, if $G / N$ has finite exponent then it is of finite rank, as was the case for $L / M$. The result follows.

It is clear that the hypotheses of Theorems 2 and 3 are inherited by subgroups and by homomorphic images. We also observe that if a group $G$ satisfies the weak maximal or weak minimal condition for abelian subgroups of infinite rank then every abelian subgroup of $G$ is easily seen to have finite rank. By the remarks in the introduction we may therefore state the following.

LEMmA 1. Let $G$ be a group which satisfies either the weak maximal or the weak minimal condition for subgroups of infinite rank. If $G$ is locally finite or locally nilpotent or hyperabelian then $G$ has finite rank.

Our next prerequisite is quite trivial.

LEMMA 2. Let $G$ be a group that is the ascending union of subgroups $F_{0}<F_{1}<\ldots$, and for each $i \geq 1$ let $N_{i}$ be a normal subgroup of $F_{i}$. Let $N$ be the subgroup generated by the $N_{i}$, and suppose that $N_{i+1} \cap F_{i} \leq N_{i}$ for each $i$. Then $N \cap F_{i}=N_{1} \ldots N_{i}$ for each $i$.

Proof. Firstly we note that $N_{j}$ is normalized $N_{i}$ whenever $i \leq j$, and so the subgroup generated by the $N_{i}$ is indeed their product. Fix $i$ and let $j$ be greater than $i$. Then $\left(N_{1} \ldots N_{j}\right) \cap F_{i}=\left(N_{1} \ldots N_{j}\right) \cap F_{j-1} \cap F_{i}=\left(\left(N_{1} \ldots N_{j-1}\right)\left(N_{j} \cap F_{j-1}\right)\right) \cap$ $F_{i}=\left(N_{1} \ldots N_{j-1}\right) \cap F_{i}$, since $N_{j} \cap F_{j-1} \leq N_{j-1}$. Repeating this argument as often as necessary we obtain $\left(N_{1} \ldots N_{j}\right) \cap F_{i}=N_{1} \ldots N_{i}$, and the result follows easily.

Proof of Theorem 2. We may suppose that $G$ is countable and, by Theorem 1, locally soluble. Assuming the result false, we may further suppose that $G$ has infinite rank but that every subgroup of infinite index in $G$ has finite rank. Let $K$ be the subgroup generated by all normal subgroups $N$ of $G$ that have finite rank. Each such $N$ has an ascending characteristic abelian series and so $K$ is hyperabelian and hence, by Lemma 1 , of finite rank. Factoring, we may assume that $K=1$, so every nontrivial normal subgroup of $G$ has infinite rank and hence finite index in $G$. Let $R$ denote the finite residual of $G$. If $R$ is nontrivial then $G / R$ is finite and $R$ is a minimal normal subgroup of $G$. But a chief factor of a locally soluble group is abelian [11, 12.5.1], and we easily obtain the contradiction that $G$ is finite. So $G$ is residually finite.

Write $G$ as an ascending union of finitely generated subgroups $F_{i}$, let $T_{i}$ be the torsion radical of $F_{i}$ and $H_{i} / T_{i}=F i t t\left(F_{i} / T_{i}\right)$, for each $i$. Observe that each $F_{i}$ has finite rank (for example, by Lemma 1). If there is a bound for the ranks of the $H_{i} / T_{i}$ then we deduce from Proposition 2 that $F_{i}^{(d)} \leq T_{i}$ for all $i$, where $d$ is some positive integer independent of $i$, so that $G^{(d)}$ is periodic and hence locally finite. By Lemma 1 $G^{(d)}$ has finite rank and is therefore trivial, so $G$ is soluble and Lemma 1 gives a contradiction. Thus the ranks of the $H_{i} / T_{i}$ are unbounded. Let $H=H_{1} H_{2} \ldots$ and, for each $i$, let $L_{i}=\left\langle H_{i}, H_{i+1} \ldots\right\rangle$. Each $L_{i}$ has infinite rank and therefore has finite index 
in $G$. It is routine to show that $H_{i+1} \cap F_{i} \leq H_{i}$ for all $i$, and so $H \cap F_{i}=H_{1} \ldots H_{i}$, by Lemma 2 . In the same manner we have $L_{i} \cap F_{i}=H_{i}$ (for example, by relabelling so that the sequence begins at $F_{i}$ ). Thus $F_{i} / H_{i}$ is finite for all $i$. Furthermore, each $F_{i}$ is minimax and residually finite, and so $T_{i}$ is finite for all $i$, and thus $F_{i}$ is finite-bynilpotent-by-finite and hence nilpotent-by-finite. We may now appply Theorem 4 to obtain the contradiction that $G$ has finite rank, and this completes the proof.

There is one further result that we shall need for the proof of Theorem 3; it appears as [8, Lemma 2] and is recorded here for convenience. In any case it is easy to prove, using the fact that the Hirsch length of a soluble minimax group is finite.

LEMMA 3. Let $G$ be a group that is locally (soluble of finite rank) and suppose that $G$ is residually (torsion-free nilpotent). Then $G$ is locally nilpotent.

Proof of Theorem 3. Again we may assume that $G$ is countable and locally soluble, also that $G$ has no nontrivial normal subgroups of finite rank. If $N$ is a nontrivial normal subgroup of $G$ then $N$ has infinite rank and so $G / N$ satisfies the weak maximal condition for all subgroups and is therefore soluble minimax [16], so the finite residual of $G / N$ is radicable (or "divisible") nilpotent, by [10, Theorem 9.31]. The intersection $V$ of all nontrivial normal subgroups $N$ of $G$ is trivial, for if not then $V$ is a chief factor of $G$ and hence abelian and therefore of finite rank, a contradiction. We deduce from this that the finite residual $R$ of $G$ is residually radicable nilpotent.

Suppose first that $R$ is nontrivial. If $S$ is a proper normal subgroup of finite index in $R$ then $1 \neq U:=R^{m} \leq S$ for some positive integer $m$, and $G / U$ is minimax, so its finite residual $R / U$ is radicable, a contradiction. Since $R$ is also a counterexample to the statement of the theorem we may assume that $G$ itself has no proper subgroups of finite index; thus $G / N$ is minimax and radicable nilpotent for all nontrivial normal subgroups $N$. Let $W$ be the intersection of all nontrivial normal subgroups $M$ of $G$ such that $G / M$ is torsion-free. Each such $G / M$ is a torsion-free locally nilpotent group of finite rank and hence nilpotent, and Lemma 3 applies to give us $G / W$ locally nilpotent and therefore nilpotent (as $W \neq 1$, by Lemma 1). If $L$ is a $G$-invariant subgroup of $W$ with $G / L$ radicable nilpotent and minimax then the torsion subgroup $W / L$ of $G / L$ is also radicable [10, Theorem 9.23] and hence abelian. Since the intersection of all such $L$ is trivial we deduce that $W$ is abelian, and we have a contradiction as before. Thus $G$ is residually finite.

Now $G$ is the ascending union of finitely generated soluble minimax subgroups $F_{0}<F_{1}<\ldots$, where each $F_{i}$ has finite torsion radical $T_{i}$ since $G$ is residually finite. Let $H_{i} / T_{i}=\operatorname{Fitt}\left(F_{i} / T_{i}\right)$ and let $K_{i} / H_{i}$ be a normal free abelian subgroup of finite index in $F_{i} / H_{i}$. If there is an upper bound for the ranks of the $H_{i} / T_{i}$ then we may apply Proposition 2 to deduce that $G^{(d)}$ is locally finite for some positive integer $d$, and then Lemma 1 gives the contradiction that $G$ has finite rank. Thus $H:=\left\langle H_{1}, H_{2} \ldots\right\rangle$ has infinite rank.

Suppose next that there is an upper bound $s$ for the ranks of the factors $K_{i} / H_{i}$. Again by Proposition 2, there is a positive integer $m$ such that, for each $i,\left(\left(F_{i}\right)^{m}\right)^{\prime}$ is finite modulo $H_{i}$. Thus $\left(G^{m}\right)^{\prime}$ is locally (finite-by-nilpotent-by-finite) and hence locally (nilpotent-by-finite). Theorem 4 and Lemma 1 now give the contradiction that $G$ has finite rank, and so the ranks of the $K_{i} / H_{i}$ are unbounded. By passing to a subsequence of the $F_{i}$ if necessary we may assume that, for all $i$, the rank of $K_{i+1} / H_{i+1}$ is greater than that of $F_{i}$. If $\left|H F_{i+1}: H F_{i}\right|$ is finite for some $i$ then, using Lemma 2, we have $\left|H F_{i+1}: H F_{i}\right|=\left|F_{i+1}: F_{i}\left(H \cap F_{i+1}\right)\right|=\left|F_{i+1}: F_{i}\left(H_{1} \ldots H_{i+1}\right)\right|=\left|F_{i+1}: F_{i} H_{i+1}\right|$, 
which is infinite. By this contradiction, each index in the chain $H F_{1}<H F_{2}<\ldots$ is infinite, and the result follows.

For the proof of Theorem 4 we need two more preliminary results. It is well-known that if $X$ is a polycyclic-by-finite group and $Y$ is a subgroup of $X$ then the intersection of all subgroups of finite index in $X$ that contain $Y$ is exactly $Y$. (For the case where $X$ is polycyclic, see $[\mathbf{1 1}, 5.4 .16]$; the same proof holds in the polycyclic-by-finite case.) Thus, if $W$ is a normal subgroup of finite index in $Y$ then there is a subgroup $M$ of finite index in $X$ with $M \cap Y=W$ (as may be seen by considering a transversal to $W$ in $Y$ ). Passing to the core of $M$ in $X$, we see that there is a normal subgroup $N$ of finite index in $X$ such that $N \cap Y \leq W$. For finitely generated nilpotent-by-finite groups we also have the following.

LEMMA 4. Let $X$ be a finitely generated nilpotent-by-finite group and let $U$ be a normal torsion-free nilpotent subgroup of finite index in $X$ (such always exists). Let $Y$ be a subgroup of infinite index in $X$ and let $n$ be a positive integer. Then there is an $X$-invariant subgroup $V$ of finite index in $U$ such that $|U: U \cap Y V|(=|U: V(U \cap Y)|)$ is divisible by $n$.

Proof. Let $W=Y \cap U$, which has infinite index in $U$. If there is a normal subgroup $M$ of finite index in $U$ such that $|U: M W|$ is divisible by $n$ then $V:=\operatorname{Core}_{X}(M)$ has finite index in $U$ and $|U: U \cap Y V|=|U: V W|$ is also divisible by $n$, and we are done. Let $Z$ be the centre of $U$; if $W Z / Z$ has infinite index in $U / Z$ then an easy induction gives such an $M$, so suppose that $|U: W Z|$ is finite. Then $W Z / W$ is finitely generated abelian and infinite, so there is a subgroup $S / W$ of index $n$ in $W Z / W$. Setting $M=$ Core $_{U}(S)$ we have the result.

Our other requirement is a trivial one, and we omit the proof.

LEMma 5. Let $X$ be a group, $\pi$ a set of primes, and let $M, N$ be normal subgroups of $X$ such that $M \leq N$ and $N / M$ is a finite $\pi$-group. Also, let $S \leq R \leq X$, where $|R: S|$ is a finite $\pi$-number. Then $|R N: S M|$ is a finite $\pi$-number.

Proof of Theorem 4. We may suppose that $G$ is countable and hence that $G$ is an ascending union of finitely generated subgroups $F_{i}$ of increasing Hirsch length. For each $i$ there is a normal torsion-free nilpotent subgroup $K_{i}$ of finite index in $F_{i}$, and (since $F_{i}$ is polycyclic-by-finite) we may choose the $K_{i}$ so that $K_{i+1} \cap F_{i} \leq K_{i}$ for all $i$. Let $p_{1}$ be a prime and let $V_{1}$ be an arbitrary $F_{1}$-invariant subgroup of finite index in $K_{1}$ such that $n_{1}:=\left|K_{1}: V_{1}\right|$ is divisible by $p_{1}$ - such exists, setting $Y=1$ in Lemma 4. Let $p_{2}$ be a prime not dividing $n_{1}$. Again by Lemma 4 , there is an $F_{2}$-invariant subgroup $V_{2}$ of finite index in $K_{2}$ such that $n_{2}:=\left|K_{2}: K_{2} \cap V_{1} V_{2}\right|$ is divisible by $n_{1}^{2} p_{2}$, and $V_{2}$ may be chosen so that $V_{2} \cap F_{1} \leq V_{1}$. Continue, so that at the $(i+1) s t$ step (and with the obvious notation) there is an $F_{i+1}$-invariant subgroup $V_{i+1}$ of finite index in $K_{i+1}$ such that $V_{i+1} \cap F_{i} \leq V_{i}$ and such that $n_{i+1}:=\left|K_{i+1}: K_{i+1} \cap\left(V_{1} \ldots V_{i+1}\right)\right|$ is divisible by $n_{i}^{2} p_{i+1}$. Let $H=\left\langle V_{i}: i \geq 1\right\rangle$ (which is the product of the $V_{i}$ since $V_{i} \triangleleft F_{i}$ for each $i$ ), and note that $H \cap F_{i}=V_{1} \ldots V_{i}$ for each $i$, by Lemma 2 and the choice of the $V_{i}$.

Now let $l$ be a fixed positive integer and (with the above notation) let $\pi_{1}=$ $\left\{p_{1}, \ldots, p_{l-1}\right\}(=\phi$ if $l=1), \pi_{2}=\pi_{1} \cup\left\{p_{l}\right\}$. For each $i \geq 1$, let $J_{i} / V_{i}$ denote the $\pi_{1}$-radical of the finite nilpotent group $K_{i} / V_{i}$, and let $L_{i} / V_{i}$ denote the $\pi_{2}$-radical. Certainly $J_{i}$ and $L_{i}$ are normal in $F_{i}$, and $J_{i} \leq L_{i}$.

Claim (i) For each $i,\left|L_{1} \ldots L_{i}: J_{1} \ldots J_{i}\right|$ is a (finite) power of $p_{l}$, and $\mid J_{1} \ldots J_{i}$ : $V_{1} \ldots V_{i} \mid$ is a finite $\pi_{1}$-number. 
First note that the result holds for $i=1$, by the definition of $J_{1}$ and $L_{1}$. Supposing true for $i-1$ we may apply Lemma 5 (with $(M, N),(S, R)=\left(J_{i}, L_{i}\right),\left(V_{i}, J_{i}\right)$ respectively) to establish the claim by induction.

Claim (ii) There is no upper bound for either of the indices $\left|L_{1} \ldots L_{i}: J_{1} \ldots J_{i}\right|$, $\left|J_{1} \ldots J_{i}: V_{1} \ldots V_{i}\right|$.

First consider $\left|L_{1} \ldots L_{i}: J_{1} \ldots J_{i}\right|$, which we know is a power of $p_{l}$ (for all $i$ ). Let $k$ be a positive integer. By construction, $\left|K_{i}: K_{i} \cap\left(V_{1} \ldots V_{i}\right)\right|$ is divisible by $p_{l}^{k}$ for all sufficiently large $i$. For such an $i$, consider the subnormal series $V_{i} \leq K_{i} \cap\left(V_{1} \ldots V_{i}\right) \leq$ $K_{i} \cap\left(J_{1} \ldots J_{i}\right) \leq K_{i} \cap\left(L_{1} \ldots L_{i}\right) \leq K_{i}$, where of course $K_{i} / V_{i}$ is a finite nilpotent group. Since $K_{i} / L_{i}$ is a $p_{l}^{\prime}$-group, the index $\left|K_{i}: K_{i} \cap\left(L_{1} \ldots L_{i}\right)\right|$ is not divisible by $p_{l}$. Also, since $\left|J_{1} \ldots J_{i}: V_{1} \ldots V_{i}\right|$ is a $\pi_{1}$-number (by Claim (i)), so is $\mid K_{i} \cap\left(J_{1} \ldots J_{i}\right)$ : $K_{i} \cap\left(V_{1} \ldots V_{i}\right) \mid$. It follows that $\left|K_{i} \cap\left(L_{1} \ldots L_{i}\right): K_{i} \cap\left(J_{1} \ldots J_{i}\right)\right|$ is divisible by $p_{l}^{k}$, and therefore so is $\left|L_{1} \ldots L_{i}: J_{1} \ldots J_{i}\right|$. The argument for $\left|J_{1} \ldots J_{i}: V_{1} \ldots V_{i}\right|$ is similar: let $m$ be an arbitrary $\pi_{1}$-number and choose $i$ so that $\left|K_{i}: K_{i} \cap\left(V_{1} \ldots V_{i}\right)\right|$ is divisible by $m$, then use the fact that $\left|K_{i}: K_{i} \cap\left(J_{1} \ldots J_{i}\right)\right|$ is a $\pi_{1}^{\prime}$-number.

We are now in a position to complete the proof of the theorem. With $J$ and $L$ as above we see that $J=\cup_{i=1}^{\infty} H\left(J_{1} \ldots J_{i}\right)$ (where each term is indeed a subgroup of $G$ ). But $\left|H\left(J_{1} \ldots J_{i}\right): H\right|=\left|\left(J_{1} \ldots J_{i}\right):\left(J_{1} \ldots J_{i}\right) \cap H\right|=\left|\left(J_{1} \ldots J_{i}\right):\left(J_{1} \ldots J_{i}\right) \cap F_{i} \cap H\right|=$ $\left|\left(J_{1} \ldots J_{i}\right):\left(V_{1} \ldots V_{i}\right)\right|$, and this increases with $i$ and so $|J: H|$ is infinite. Similarly $|L: J|$ is infinite, and by successive application of these facts we obtain an infinite chain of subgroups $H=H_{0}<H_{1}<H_{2}<\ldots$, where each index $\left|H_{i+1}: H_{i}\right|$ is infinite (and, in addition, every element of $H_{i+1}$ has a nonzero $p_{i}$-power in $H_{i}$ ). On the other hand, by letting $K=\left\langle K_{i}: i \geq 1\right\rangle$ and taking direct complements $M_{i} / V_{i}, N_{i} / V_{i}$ in $K_{i} / V_{i}$ for subgroups such as $J_{i} / V_{i}$ and $L_{i} / V_{i}$ and forming the subgroups of $K$ generated by the $M_{i}, N_{i}$ respectively, we can obtain a descending series of subgroups of $K$, each of infinite index in the preceding one and each of infinite rank. We omit the details. It is clear from the construction that $I_{G}(H)=G=I_{G}(K)$, and the proof is complete.

It remains only to establish Theorem 5. Our final lemma is again easy to prove (although our proof appeals to another substantial result on groups of finite rank).

LEMMA 6. Let G be a locally soluble-by-finite group with just finitely many conjugacy classes of subgroups. Then $G$ is finite.

Proof. Clearly there is an integer $r$ such that every finitely generated subgroup of $G$ is $r$-generated, that is, $G$ has rank at most $r$. By [3] $G$ is almost locally soluble and hence almost soluble, since there is a finite upper bound for the derived length of soluble subgroups. Since our hypothesis is inherited by homomorphic images we may assume by induction that $G$ is abelian-by-finite. But the hypothesis is also inherited by normal subgroups of finite index, and since an abelian group with this property is clearly finite, the result follows.

Proof of Theorem 5. Suppose the result false, so that $G$ has exactly $k$ conjugacy classes of subgroups of infinite rank, for some positive integer $k$; note that every homomorphic image of $G$ has at most $k$ such conjugacy classes. If $N$ is a normal subgroup of $G$ of infinite rank then $G / N$ has just finitely many conjugacy classes of subgroups and is therefore finite, by Lemma 6. Let $R$ denote the finite residual of $G$. As $G$ has only finitely many normal subgroups of finite index, $G / R$ is finite, and since $R$ inherits the hypotheses on $G$ (with perhaps a different " $k$ ") we may assume that $R=G$, that is, $G$ has no proper subgroups of finite index. Thus every proper normal 
subgroup of $G$ has finite rank. If $G^{\prime}<G$ then $G / G^{\prime}$ has just finitely many subgroups of infinite rank and hence no such subgroups (as an abelian group of infinite rank has a proper subgroup of infinite rank), and we obtain the contradiction that $G$ has finite rank. Hence $G$ is perfect.

Let $N$ be a proper normal subgroup of $G$. As $N$ has finite rank it has a locally soluble radical $L$ of finite index, and since $N / L$ is finite its centralizer in $G$ has finite index and hence equals $G$; thus $N$ is locally soluble and has an ascending characteristic abelian series. The subgroup $K$ generated by all such $N$ is hyperabelian and locally of finite rank, so it is locally soluble. Assume first that $K=G$ and let $A$ be an arbitrary $G$-invariant abelian factor that has finite rank and is either torsion-free or a (finite) elementary abelian $p$-group for some prime $p$. By the result of Zassenhaus referred to earlier, some finite term of the derived series of $G$ centralizes $A$, so $A$ is central since $G$ is perfect. Thus every proper normal subgroup of $G$ is contained in the hypercentre, giving $G$ hypercentral and hence trivial (as it is perfect), a contradiction. Hence $K<G$ and, factoring, we may assume that $G$ is simple.

Next, suppose that every locally finite group satisfying our hypotheses has finite rank; so $G$ is not locally finite. Choose an element $x$ of infinite order in $G$ and let $U$ be a countable subgroup of $G$ that contains $x$ and has infinite rank. Since $G$ is simple we may embed $U$ in a countable simple subgroup of $G$ [6, Theorem 4.4], so we may assume that $U$ is itself simple. Write $U$ as the ascending union of finitely generated subgroups $F_{i}$ and let $S_{i}$ be the soluble radical of $F_{i}$ for each $i$, so that $F_{i} / S_{i}$ is finite. Let $R=\left\langle S_{i}: i \geq 1\right\rangle$ and let $X_{i}=\left\langle R, F_{i}\right\rangle$ for each $i$. Then $X_{i}=R F_{i}$, since $F_{i}$ normalizes $S_{j}$ for all $j \geq i$, and $\left|X_{i}: R\right|$ is clearly finite. If $R$ has infinite rank then the $X_{i}$ fall into finitely many conjugacy classes, and so there is an upper bound $n$ for the index in $X_{i}$ of its locally soluble radical. Then $X_{i}^{n !}$ is locally soluble for all $i$, so $U^{n !}$ is locally soluble. But $U$ is simple and infinite, so it is not locally soluble, and it follows that $U^{n !}=1$ and $U$ is locally finite, a contradiction. Thus $R$ has finite rank and so, by [10, Lemma 10.39], $R^{(d)}$ is locally finite, for some positive integer $d$. Now $R / R^{(d)}$ has finite Hirsch length $r$, say, that is, the sum of the 0 -ranks of a finite normal abelian series of $R / R^{(d)}$ is $r$. Thus the Hirsch length of any soluble section of each $F_{i}$ is at most $r$. Let $T_{i}$ be the torsion radical of $F_{i}, H_{i} / T_{i}=\operatorname{Fitt}\left(F_{i} / T_{i}\right)$, and let $K_{i} / H_{i}$ be a normal free abelian subgroup of finite index in $F_{i} / H_{i}$. Then $K_{i} / T_{i}$ has rank at most $r$, and Proposition 1 applies to give us a finite normal series for $U$ with factors periodic or nilpotent. But $U$ is simple and hence locally finite, a contradiction.

It remains only to deal with the case where $G$ is locally finite. By [14] $G$ has an abelian subgroup $A$ of infinite rank, and since $A$ in turn has an infinite descending chain of subgroups of infinite rank it follows easily that there is a subgroup $C$ of $A$ such that $C^{g}$ is a proper subgroup of $C$ for some $g$ in $G$. But $g$ has finite order $n$, say, and since $C^{g^{i}}<C^{g^{i-1}}$ for each positive integer $i$ we obtain the contradiction that $C=C^{g^{n}}<C$. This completes the proof.

\section{REFERENCES}

1. R. Baer and H. Heineken, Radical groups with finite abelian subgroup rank, Illinois $J$. Math. 16 (1972), 533-580.

2. R. Brandl, S. Franciosi and F. de Giovanni, Groups with finitely many conjugacy classes of non-normal subgroups, Proc. Roy. Irish Acad. Sect A 95 (1995), 17-27.

3. N. S. Černikov, A theorem on groups of finite special rank, Ukrain. Mat. Zh. 42 (1990), 962-970 (Russian), English transl. in Ukrainian Math. J. 42 (1990), 855-861. 
4. M. R. Dixon, M. J. Evans and H. Smith, Locally (soluble-by-finite) groups of finite rank, J. Algebra 182 (1996), 756-769.

5. M. R. Dixon, M. J. Evans and H. Smith, Locally (soluble-by-finite) groups with the weak minimal condition on non-nilpotent subgroups, J. Algebra 249 (2002), 226-246.

6. O. H. Kegel and B. A. F. Wehrfritz, Locally finite groups (North-Holland, 1973).

7. L. A. Kurdachenko and H. Smith, Groups with the weak maximal condition for nonsubnormal subgroups, Annali di Mat. pura ed applicata 4 (1997), 299-312.

8. L. A. Kurdachenko and H. Smith, Groups in which all subgroups of infinite rank are subnormal, Glasgow Math. J. 46 (2004), 83-90.

9. Yu. I. Merzljakov, Locally soluble groups of finite rank, Algebra i Logika 3(1964), 5-16; erratum, 8 (1969), 686-690.

10. D. J. S. Robinson, Finiteness conditions and generalized soluble groups (Springer-Verlag, 1972).

11. D. J. S. Robinson, A course in the theory of groups (Springer-Verlag, 1982).

12. H. Smith, Groups with finitely many conjugacy classes of subgroups of large derived length, Bollettino U.M.I. 7 (1995), 167-175.

13. H. Smith, Groups with few non-nilpotent subgroups, Glasgow Math. J. 39 (1997), 141151.

14. V. P. Šunkov, On locally finite groups of finite rank, Algebra and Logic 10 (1971), $127-142$.

15. B. A. F. Wehrfritz, Infinite linear groups (Springer-Verlag, 1973).

16. D. I. Zaičev, Theory of minimax groups, Ukrainian Math. J. 23 (1971), 536-542. 